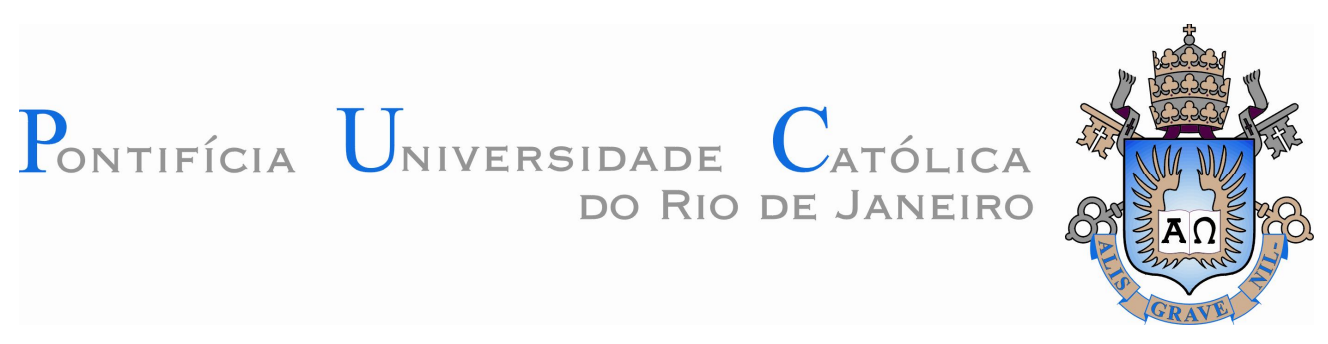

Elvis Yuri Mamani Vargas

\title{
O Método Híbrido dos Elementos de Contorno com Base em Funções de Tensão de Westergaard Generalizadas
}

\section{Dissertação de Mestrado}

Dissertação apresentada como requisito parcial para obtenção do título de Mestre pelo Programa de PósGraduação em Engenharia Civil do Departamento de Engenharia Civil da PUC-Rio.

Orientador: Prof. Ney Augusto Dumont

Rio de Janeiro 


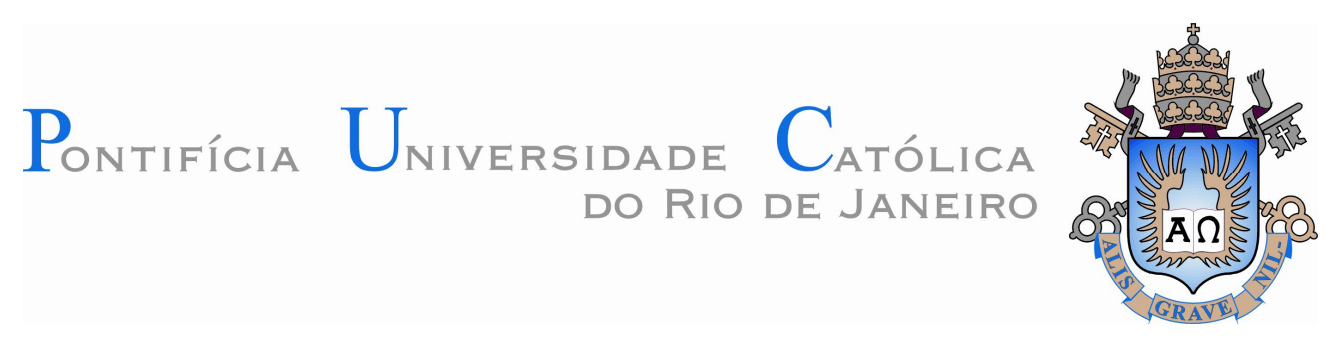

Elvis Yuri Mamani Vargas

\title{
O Método Híbrido dos Elementos de Contorno com Base em Funções de Tensão de Westergaard Generalizadas
}

Dissertação apresentada como requisito parcial para obtenção do título de Mestre pelo Programa de PósGraduação em Engenharia Civil da PUC-Rio. Aprovada pela Comissão Examinadora abaixo assinada.

\author{
Prof. Ney Augusto Dumont \\ Orientador \\ Departamento de Engenharia Civil ï PUC-Rio \\ Prof. Raul Rosas e Silva \\ Departamento de Engenharia Civil ï PUC-Rio
}

Prof. Euclides de Mesquita Neto Universidade Estadual de Campinas

Prof. Rubens de Oliveira Universidade Federal de Juiz de Fora

Prof. Alexandre Antônio de Oliveira Lopes Petrosoft Design

Prof. José Eugênio Leal Coordenador Setorial do Centro Técnico Científico ï PUC-Rio

Rio de Janeiro, 25 de fevereiro de 2011. 
Todos os direitos reservados. É proibida a reprodução total ou parcial do trabalho sem autorização da universidade, do autor e do orientador.

\section{Elvis Yuri Mamani Vargas}

Graduou-se em Engenharia Civil no Departamento de Engenharia Civil da UNSAAC (Universidad Nacional de San Antonio Abad del Cusco ï Perú), em 2005. Em 2009 iniciou o curso de Mestrado em Engenharia Civil na PUCï Rio, na área de Estruturas, atuando na linha de pesquisa do método híbrido dos elementos de contorno.

Ficha Catalográfica

Vargas, Elvis Yuri Mamani

O Método Híbrido dos Elementos de Contorno com Base em Funções de Tensão de Westergaard Generalizadas / Elvis Yuri Mamani Vargas; orientador: Ney Augusto Dumont. ï Rio de Janeiro: PUC, Departamento de Engenharia Civil, 2011.

117 f.: il. (cor); $29,7 \mathrm{~cm}$

1. Dissertação (mestrado) - Pontifícia Universidade Católica do Rio de Janeiro, Departamento de Engenharia Civil.

Incluí referências bibliográficas.

1. Engenharia Civil - Teses. 2. Elementos de contorno. 3. Métodos híbridos. 4. Soluções fundamentais. 5. Mecânica da fratura. 6. Funções de tensão de Westergaard. 7. Fator de intensidade de tensão. I. Dumont, Ney A. (Ney Augusto). II. Pontifícia Universidade Católica do Rio de Janeiro - Departamento de Engenharia Civil. III. Título.

CDD: 624 
Aos meus pais Vidal e Rosa, pelo inesgotável amor, apoio e estímulo que me ofereceram nos momentos mais difíceis.

À minha irmã Chris pela compressão e ajuda que sempre me ofereceu. Ao Peru, pelo legado das culturas antigas. 


\section{Agradecimentos}

Ao Deus por ter me concedido a vida.

Ao meu orientador Professor Ney Augusto Dumont pela orientação, confiança, conhecimento transmitido e sua disposição prestara na orientação deste trabalho.

Ao professor Alexandre Antônio de Oliveira Lopes pela confiança, estímulo, conhecimento transmitido, conselhos e sua amizade.

Ao CNPq e à PUC-Rio, pelos auxílios concedidos, sem os quais este trabalho não poderia ter sido realizado.

Aos professores da PUC-Rio, pelos ensinamentos transmitidos durante estes dois anos.

Aos professores da UNSAAC-Peru, pelos ensinamentos básicos da engenharia transmitidos durante a graduação.

Aos meus amigos Fernando, Eliot, Jorge, Gino (a fera), Júlio (jacaré), Evelin, Roxana, os amigos das peladas dos sábados e tantos outros que não citei por todo apoio, paciência e compreensão que tornarem esta jornada mais agradável.

Aos meus pais e irmãos pela educação, atenção e carinho de todas as horas.

A todos os amigos e familiares que de uma forma ou de outra me estimularam ou me ajudaram.

Ao Brasil país maravilhoso e a sua gente que sempre me fez sentir em casa. 


\section{Resumo}

Vargas, Elvis Yuri Mamani; Dumont, Ney Augusto. O Método Híbrido dos Elementos de Contorno com Base em Funções de Tensão de Westergaard Generalizadas. Rio de Janeiro, 2011. 117p. Dissertação de Mestrado - Departamento de Engenharia Civil, Pontifícia Universidade Católica do Rio de Janeiro.

Apresenta-se uma formulação particular do método híbrido dos elementos de contorno para a análise de problemas planos de potencial e de elasticidade que, apesar de completamente geral, é apropriada a aplicações de mecânica da fratura. Funções do tipo de Westergaard são usadas como soluções fundamentais, Em uma generalização de uma proposta inicialmente feita por Tada et al. A formulação leva a conceitos de elementos de contorno de deslocamentos semelhante à apresentada por Crouch e Starfield, mas em um contexto variacional que permite interpretações mecânicas bem simples das equações matriciais resultantes. Problemas de topologia geral podem ser modelados, como no caso de domínios infinitos ou multiplamente conexos. A formulação, que é diretamente aplicável a placas com entalhes ou trincas curvas externas ou internas, permite a descrição adequada de altos gradientes de tensão e é uma ferramenta simples de avaliação de fatores de intensidade de tensão, com o que se podem verificar numericamente conceitos estabelecidos por Rice em 1968. Esta dissertação tem foco na fundamentação matemática da formulação para problemas de potencial e de elasticidade. Apresenta-se a implementação da formulação e são discutidos vários exemplos numéricos de validação.

\section{Palavras-chave}

Elementos de contorno; métodos híbridos; soluções fundamentais; mecânica da fratura; Funções de tensão de Westergaard; fator de intensidade de tensão. 


\section{Abstract}

Vargas, Elvis Yuri Mamani; Dumont, Ney Augusto (Advisor). The Hybrid Boundary Element Method Based on Generalized Westergaard Stress Functions. Rio de Janeiro, 2011. 117p. MSc. Dissertation - Departamento de Engenharia Civil, Pontifícia Universidade Católica do Rio de Janeiro.

A particular implementation of the hybrid boundary element method is presented for the two dimensional analysis of potential and elasticity problems, which although general in concept, is suited for fracture mechanics applications. Generalized Westergaard stress functions, as proposed by Tada et al in 1993, are used as the problemó fundamental solutions. The proposed formulation leads to displacement-based concepts that resemble those presented by Crouch and Starfield, although in a variational framework that leads to matrix equations with sound mechanical meanings. Problems of general topology, such as in the case of unbounded and multiply-connected domains, may be modeled. The formulation, which is directly applicable to notches and generally curved, internal or external cracks, is specially suited for the description of the stress field in the vicinity of crack tips and is an easy means of evaluating stress intensity factors and of checking some basic concepts laid down by Rice in 1968. This dissertation focuses on the mathematical fundamentals of the formulation. Several validating numerical examples are presented.

\section{Keywords}

Boundary elements; hybrid methods; fundamental solutions; fracture mechanics; Westergaard stress functions; stress intensity factors. 


\section{Sumário}

1 INTRODUÇÃO 20

1.1. Considerações iniciais 20

1.2. Objetivos e delimitações 22

1.3. Estrutura da dissertação 23

2 FUNDAMENTOS DA MECÂNICA DA FRATURA 25

2.1. Mecânica da Fratura 25

2.2. Concentração de Tensões 26

2.3. Balanço de energia de Griffith 28

2.4. Fatores de Intensidade de Tensão 29

2.5. Função de Tensão Complexa de Westergaard 33

2.6. Integral J 39

3 MÉTODO HÍBRIDO DOS ELEMENTOS DE CONTORNO 41

3.1. Teoria da Elasticidade 42

3.2. Solução Fundamental de Kelvin 44

3.3. Potencial de Hellinger-Reissner 46

3.4. Estabelecimento da formulação 48

3.5. Transformações entre o sistema interno e externo de coordenadas 52

3.6. Obtenção de tensões e deslocamento no domínio 54

3.7. Caso particular de estruturas estaticamente

determinadas $\quad 55$

4 FUNCÃO DE TENSÃO DE WESTERGAARD GENERALIZADA APLICADA A PROBLEMAS DE POTENCIAL 2D 57

4.1. Potencial e fluxos para uma trinca genérica 58

4.2. Estabelecimento da função de tensão para uma trinca semi-elíptica 
4.3. Considerações das singularidades e descontinuidades

4.3.1. Expansão da função de tensão $\Phi$ em torno à origem

4.3.2. Expansão da derivada da função de tensão $\Phi^{\prime}$ em torno à origem

4.3.3. Expansão da derivada da função de tensão $\Phi^{\prime}$ em torno à ponta

4.4. Formulação para um domínio finito

4.4.1. Exemplos

4.5. Formulação para uma trinca em um meio infinito

4.5.1. Exemplos

4.6. Formulação para uma trinca em um domínio finito

4.6.1. Exemplos

4.7. A Integral J no cálculo de fatores de intensidade de tensão

4.7.1. A integral J para problemas de potencial

4.7.2. Relação da integral $\mathrm{J}$ com o fator de intensidade de tensão

4.7.3. Exemplos

4.8. Aproximações polinomiais para o cálculo do fator de intensidade de tensão

4.8.1. Exemplos

5 FUNCÃO DE TENSÃO DE WESTERGAARD GENERALIZADA APLICADA A PROBLEMAS DE ELASTICIDADE 2D

5.1. Deslocamentos e tensões para uma trinca genérica

5.1.1. Modo I de trincamento

5.1.2. Modo II de trincamento

5.2. Deslocamentos para a ação combinada de duas trincas

102

5.3. Estabelecimento da função de tensão para uma trinca semi-elíptica

5.4. Considerações das singularidades e descontinuidades

5.5. Superposição de efeitos de duas trincas semi-elípticas 
6 CONCLUSÕES E SUGESTÕES

6.1. Conclusões

111

6.2. Sugestões para trabalhos futuros

113

7 REFERÊNCIAS BIBLIOGRÁFICAS

114 


\section{Lista de figuras}

Figura 2.1 - Entalhe circular em uma placa plana infinita 27

Figura 2.2 - Entalhe elíptico em uma placa plana infinita 27

Figura 2.3 - Modelo de Griffith para uma trinca 28

Figura 2.4 - Definição do sistema de coordenadas para a trinca 30

Figura 2.5 - Modos fundamentais de trincamento 31

Figura 2.6 - Trinca em uma placa infinita submetida a tensão biaxial 33

Figura 2.7 - Representação gráfica da função de tensão de

Westergaard modificada $\quad 36$

Figura 2.8 - Contorno arbitrário em torno da ponta de uma trinca $\quad 39$

Figura 2.9 - Contorno fechado utilizado para cálculo da integral J 40

Figura 3.1 - Corpo elástico em equilíbrio 42

Figura 3.2 - Força singular aplicada em um ponto 44

Figura 3.3 - Gráfico da energia interna de deformação 47

Figura 3.4 - Sistema de coordenadas, para descrição do comportamento da estrutura 49

Figura 4.1 - Abertura semi-elíptica para uma trinca com comprimento $a=1$

Figura 4.2 - Abertura semi-elíptica para uma trinca com comprimento $a_{1}$ e rotação $\theta_{1}$

Figura 4.3 - Função $c \operatorname{sgn}($ ) da expansão em séries de $\Phi$ em torno à origem

Figura 4.4 - Abertura de um elemento de trinca formado por duas trincas semi-elípticas

Figura 4.5 - Exemplo da discretização de um contorno

$\Gamma$ com 12 elementos de trinca

Figura 4.6 - Estrutura para avaliação do potencial e dos fluxos em pontos internos, devido a uma fonte externa $F$

Figura 4.7 - Potencial $u$ ao longo da linha tracejada 
Figura 4.8 - Gradientes $q_{x}$ e $q_{y}$ ao longo da linha tracejada

A-B da Figura 4.6

Figura 4.9 - Erro do potencial $u$ e dos fluxos $q_{x}$ e $q_{y}$ ao longo

da linha tracejada A-B da Figura 4.6

Figura 4.10 - Trinca curva discretizada com $n$ elementos

Figura 4.11 - Trinca reta de comprimento $a=1$ submetida

a um fluxo remoto unitário

Figura 4.12 ï Fatores de intensidade de tensão $K_{I}$ para la trinca da Figura 4.11

Figura 4.13 - Erro dos fatores de intensidade de tensão $K_{I}$ para a trinca da Figura 4.11

Figura 4.14 - Convergência da gradiente $q_{y}$ ao longo da linha tracejada na Figura 4.11

Figura 4.15 - Erro no gradiente $q_{y}$ ao longo da linha tracejada da Figura 4.11

Figura 4.16 - Trinca curva de raio $\sqrt{2}$ submetida a um fluxo remoto unitário

Figura 4.17 - Convergência da gradiente $q_{y}$ ao longo da linha tracejada da Figura 4.16

Figura 4.18 - Trinca concêntrica em uma placa retangular submetida a um fluxo unitário

Figura 4.19 - Fatores de intensidade de tensão $K_{I}$ para a trinca da Figura 4.18

Figura $4.20-K_{I}$ a partir da Integral $J$, para varias discretizações da trinca

Figura 4.21 - $K_{I}$ a partir da Integral $J$, em função da distância da ponta da trinca ao ponto onde o caminho $\Gamma_{J}$ corta à trinca

Figura 4.22 - $K_{I}$ a partir de $J$, em função das dimensões de $\Gamma_{J}$

Figura $4.23-K_{I}$ a partir de $J$, para varias discretizações da trinca 
influenciada por um furo próximo ï ponta $\mathrm{A}$

Figura $4.24-K_{I}$ a partir de $J$, para varias discretizações da trinca influenciada por um furo próximo - ponta $B$

Figura $4.25-K_{I}$ a partir de $J$, para uma trinca com influencia de um furo próximo - varias posições do caminho $\Gamma_{J}$

Figura 4.26 - $K_{I}$ para uma trinca com a influencia de um furo próximo - varias posições do contorno $\Gamma_{J}$

Figura $4.27-K_{I}$ a partir de $J$ em função do numero de elementos discretizado de uma trinca com influencia de dois furos próximos

Figura 4.28 - $K_{I}$ a partir de $J$ em função da posição do contorno $\Gamma_{J}$, trinca reta com a influencia de dois furos próximos

Figura 4.29 - Detalhe da abertura de uma trinca, próxima a sua ponta

Figura 4.30 - Fatores de intensidade de tensão para a trinca

da Figura 4.11 a partir de aproximações polinomiais

Figura 4.31 - Erro dos fatores de intensidade de tensão para a trinca da Figura 4.11 a partir de aproximações polinomiais com três termos Figura 5.1 - Deslocamentos e tensões para o modo I de trincamento, segundo sistema local e global de coordenadas

Figura 5.2 - Deslocamentos e tensões para o modo II de trincamento, segundo sistema local e global de coordenadas

Figura 5.3 - Superposição de efeitos de duas trincas semi-elípticas

Figura 5.4 - Trinca reta de comprimento $2 a=2$ submetida a um carregamento remoto unitário

Figura 5.5 - Interpretação física do parâmetro p*

Figura 5.6 - Tensões $\sigma_{y y}$ ao longo da linha tracejada da Figura 5.4

Figura 5.7 - Estrutura para avaliação das tensões em pontos internos, devido a uma força unitária aplicada no ponto $F$

Figura 5.8 - Tensões analíticas e numéricas ao longo do segmento de linha $A B$, para uma força horizontal no ponto $(-10.25)$ 


\section{Lista de tabelas}

Tabela 2.1 - Tensões e deslocamentos para os modos I e II de trincamento no sistema cartesiano de coordenadas

Tabela 2.2 - Tensões para os modos I e II de trincamento no sistema polar de coordenadas

Tabela 2.3 - Representação gráfica das componentes de tensão $\sigma_{i j}$ nos modos I e II de trincamento para o caso particular de $a=1$

Tabela 2.4 - Representação gráfica das componentes de deslocamentos $u$ e $v$ nos modos I e II de trincamento para o caso particular de $a=1, v=0,30$ e $E=1$

Tabela 4.1 - Representação gráfica das funções $\Im(\Phi), \mathfrak{R}(\Phi)$, $\Im\left(\Phi^{\prime}\right)$ e $\mathfrak{R}\left(\Phi^{\prime}\right)$ para o caso particular de $Z=x+i y$

Tabela 4.2 - Dimensões e discretizações das arestas da placa

da Figura 4.18

Tabela 4.3 - Expressões para o cálculo dos fatores de intensidade de tensão $K_{I}$ usando aproximações polinomiais 


\section{Lista de símbolos}

\section{Caracteres latinos:}

A Ponto extremo da elipse

a Comprimento da trinca

$a_{c} \quad$ Comprimento crítico da trinca

$a_{1} \quad$ Comprimento do primeiro segmento da trinca discretizada

$a_{n+1} \quad$ Comprimento do último segmento da trinca discretizada

B Ponto extremo da elipse

$b \quad$ Comprimento do entalhe elíptico

$b_{k},\{\mathbf{b}\} \quad$ Deslocamentos do sistema interno equivalentes ao campo de deslocamentos referentes às forças de massa

$C_{i j} \quad$ Constantes arbitrárias do campo de deslocamentos referentes à solução fundamental

$C_{i j k l} \quad$ Tensor da relação constitutiva

$d_{j},\{\mathbf{d}\} \quad$ Deslocamentos nodais do sistema externo

$d_{k}^{*},\left\{\mathbf{d}^{*}\right\}$ Deslocamentos nodais equivalentes do sistema interno

E Módulo de Young, modulo de elasticidade

$E_{k l},[\mathrm{E}] \quad$ Projetor ortogonal

$f_{i j} \quad$ Função adimensional de $\theta$

$F\left(\theta^{*}, \lambda\right) \quad$ Função adimensional de $\theta^{*}$ e $\lambda$

$\bar{F}_{i},\{\bar{F}\} \quad$ Forças de massa prescritas

$F_{k l},[\mathbf{F}] \quad$ Matriz de flexibilidade do sistema interno

$G \quad$ Taxa de liberação de energia de deformação

$G_{c} \quad$ Taxa crítica de liberação de energia de deformação

$H_{k l},[\mathbf{H}] \quad$ Matriz de incidência cinemática

$\mathbf{H}_{00} \quad$ Matriz referente à solução fundamental de Kelvin no contorno externo com integração no contorno externo

$\mathbf{H}_{\mathbf{0 c}} \quad$ Matriz referente à solução fundamental de Kelvin no contorno 
externo com integração no contorno da trinca

\begin{tabular}{|c|c|}
\hline $\mathbf{H}_{\mathrm{co}}$ & $\begin{array}{l}\text { Matriz referente à solução fundamental de Westergaard no } \\
\text { contorno da trinca com integração no contorno externo }\end{array}$ \\
\hline $\mathbf{H}_{c c}$ & $\begin{array}{l}\text { Matriz referente à solução fundamental de Westergaard no } \\
\text { contorno da trinca com integração no contorno da trinca }\end{array}$ \\
\hline$i$ & Constante complexa \\
\hline$J$ & Integral J \\
\hline K & Fator de intensidade de tensão \\
\hline$K_{I, I I, I I I}$ & $\begin{array}{l}\text { Fator de intensidade de tensão para os módulos I, II e III de } \\
\text { trincamento }\end{array}$ \\
\hline$K_{t}$ & Fator de concentração de tensões \\
\hline$K_{k l},[\mathbf{K}]$ & Matriz de rigidez do sistema externo \\
\hline$k$ & Constante potencial \\
\hline$N_{L}$ & Funções de interpolação \\
\hline$p_{i},\{\mathbf{p}\}$ & Forças nodais equivalentes \\
\hline$p_{i}^{*},\left\{\mathbf{p}^{*}\right\}$ & Forças singulares \\
\hline$p_{i j}^{*},\left\{\mathbf{p}^{*}\right\}$ & $\begin{array}{l}\text { Função de transformação de forças referente à solução } \\
\text { fundamental }\end{array}$ \\
\hline$q_{i},\{\mathbf{q}\}$ & Fluxo \\
\hline$R$ & Raio do circulo \\
\hline$r$ & Módulo do vetor posição (raio) \\
\hline$t_{k},\{\mathbf{t}\}$ & $\begin{array}{l}\text { Forças nodais do sistema externo, equivalentes às forças de } \\
\text { massa }\end{array}$ \\
\hline$T_{i},\{\mathbf{T}\}$ & Forças de superfície \\
\hline $\bar{T}_{i},\{\overline{\mathbf{T}}\}$ & Forças de superfície prescritas \\
\hline$T_{i}^{*},\left\{\mathbf{T}^{*}\right\}$ & Forças de superfície referentes à solução fundamental \\
\hline$u^{I, I I}$ & $\begin{array}{l}\text { Deslocamentos segundo o eixo } x \text { de coordenadas devido aos } \\
\text { modos I e II de trincamento }\end{array}$ \\
\hline$u_{i},\{\mathbf{u}\}$ & Deslocamentos, potenciais \\
\hline $\bar{u}_{i},\{\overline{\mathbf{u}}\}$ & Deslocamentos prescritos, potenciais prescritos \\
\hline$u_{i}^{*},\left\{\mathbf{u}^{*}\right\}$ & Deslocamentos referentes à solução fundamental \\
\hline
\end{tabular}




\begin{tabular}{|c|c|}
\hline$u_{i}^{* n},\left\{\mathbf{u}^{* \mathrm{n}}\right\}$ & Deslocamentos totais referentes às forças de massa \\
\hline$u_{i}^{* p},\left\{\mathbf{u}^{* \mathbf{p}}\right\}$ & $\begin{array}{l}\text { Deslocamentos referentes à solução particular da equação de } \\
\text { equilíbrio }\end{array}$ \\
\hline$u_{i j},[\mathbf{u}]$ & Funções de interpolação de deslocamentos \\
\hline$u_{i j}^{*},\left[\mathbf{u}^{*}\right]$ & $\begin{array}{l}\text { Função de transformação de deslocamentos referente à } \\
\text { solução fundamental }\end{array}$ \\
\hline$U_{0}\left(\varepsilon_{i j}\right)$ & Densidade de energia interna de deformação \\
\hline$U_{0}^{c}\left(\sigma_{i j}\right)$ & Densidade de energia interna na forma complementar \\
\hline$U_{0}^{c^{*}}\left(\sigma_{i j}\right)$ & $\begin{array}{l}\text { Densidade de energia interna na forma complementar, } \\
\text { referente ao sistema interno }\end{array}$ \\
\hline$V_{k l},[\mathbf{V}]$ & $\begin{array}{l}\text { Matriz cujas colunas formam a base das forças singulares que } \\
\text { correspondem a forças nodais equivalentes nulas }\end{array}$ \\
\hline $\mathbf{v}$ & Espaço nulo \\
\hline$v^{I, I I}$ & $\begin{array}{l}\text { Deslocamentos segundo o eixo } x \text { de coordenadas devido aos } \\
\text { modos I e II de trincamento }\end{array}$ \\
\hline $\mathbf{v}_{\mathbf{0}}$ & $\begin{array}{l}\text { Espaço nulo decorrente da ortogonalidade a deslocamentos } \\
\text { de corpo rígido }\end{array}$ \\
\hline $\mathbf{v}_{1}$ & $\begin{array}{l}\text { Espaços nulos adicionais provenientes de cada par de nós } \\
\text { com a mesma coordenada }\end{array}$ \\
\hline$w$ & Comprimento da $p$ \\
\hline$W$ & Energia de deformação \\
\hline$W_{k l},[\mathbf{W}]$ & $\begin{array}{l}\text { Matriz cujas colunas formam a base dos deslocamentos de } \\
\text { corpo rígido }\end{array}$ \\
\hline$c_{i}$ & Coorde \\
\hline
\end{tabular}

\section{Caracteres gregos:}

$\Delta \quad$ Trabalho não recuperável associado à deformação permanente na ponta da trinca

$\Delta_{\mathrm{ij}} \quad$ Delta de Dirac

$\underline{\Phi} \quad$ Funções de tensão de Airy

$\Phi_{I, I I} \quad$ Função de tensão de Westergaard (modificada ou generalizada) para os modos I e II de trincamento 

$\Phi_{I, I I}^{\prime} \quad$ Derivada da função de tensão de Westergaard (modificada ou generalizada) para os modos I e II de trincamento
$\Phi{ }_{I, I I} \quad$ Segunda derivada da função de tensão de Westergaard $\mathrm{p}$ os modos I e II de trincamento
$\Gamma \quad$ Contorno do corpo elástico, contorno arbitrário em torno da ponta da trinca
$\Gamma_{J} \quad$ Região do contorno relacionado à Integral $J$
$\Gamma_{u} \quad$ Região do contorno onde se têm deslocamentos ou potenciais prescritos
$\Gamma_{\sigma} \quad$ Região do contorno onde se têm forças ou gradientes prescritos
$\Gamma^{*} \quad$ Contorno referente à solução fundamental
$\Gamma_{0} \quad$ Região do contorno correspondente à parte externa da superfície esférica
$\bar{\Gamma}_{0} \quad$ Região do contorno contida na superfície esférica
$\Pi \quad$ Energia potencial total
$\Pi_{g} \quad$ Forma generalizada da energia potencial total
$\Pi_{R} \quad$ Potencial de Hellinger-Heissner
$\Omega \quad$ Domínio do corpo elástico
$\Omega * \quad$ Domínio referente à solução fundamental
$\Omega_{0} \quad$ Região onde a força singular é aplicada
$\delta_{i j} \quad$ Delta de Kronecker
$\varepsilon_{i j} \quad$ Deformações
$\gamma \quad$ Trabalho necessário para formar uma nova superfície de trinca
$\eta_{j} \quad$ Cossenos diretores de um elemento de superfície
$\lambda_{i j}, \lambda_{i} \quad$ Multiplicadores de Lagrange
$\mu \quad$ Módulo de elasticidade transversal
$v \quad$ Coeficiente de Poisson
$\pi \quad$ Constante
$\theta \quad$ Ângulo do sistema de coordenadas polares
$\theta_{i} \quad$ Ângulo de rotação da trinca i em relação ao eixo positivo de $\mathrm{x}$ 


\begin{tabular}{|c|c|}
\hline$\rho$ & Raio de curvatura \\
\hline$\sigma$ & Tensão normal \\
\hline$\sigma^{\infty}$ & Tensão normal aplicada no meio infinito \\
\hline$\sigma_{c}$ & $\begin{array}{l}\text { Tensão crítica a partir da qual o crescimento da trinca é } \\
\text { instável }\end{array}$ \\
\hline$\sigma_{n}$ & Tensão normal nominal \\
\hline$\sigma_{i j}$ & Tensões normais, tensões \\
\hline$\sigma_{i j}^{I, I I}$ & $\begin{array}{l}\text { Tensões normais, tensões devido aos modo I e } \| \text { de } \\
\text { trincamento }\end{array}$ \\
\hline$\sigma_{i j}^{*}$ & Tensões referentes à solução fundamental \\
\hline$\sigma_{i j}^{* n}$ & Tensões totais referentes às forças de massa \\
\hline$\sigma_{i j}^{* p}$ & $\begin{array}{l}\text { Tensões referentes à solução particular da equação de } \\
\text { equilíbrio }\end{array}$ \\
\hline$\tau$ & Tensão cisalhante aplicada \\
\hline$\tau^{\infty}$ & Tensão cisalhante aplicada no meio infinito \\
\hline$\tau_{i j}$ & Tensões cisalhantes \\
\hline$\tau_{i j}^{I, I I}$ & Tensões cisalhantes devido aos modos I e I de trincamento \\
\hline$\xi, \eta$ & Coordenadas paramétricas \\
\hline $\mathfrak{I}()$ & Parte imaginária de um número complexo \\
\hline $\mathfrak{R}()$ & Parte real de um número complexo \\
\hline
\end{tabular}

